\title{
THE DESCENDING CHAIN CONDITION ON SOLUTION SETS FOR SYSTEMS OF EQUATIONS IN GROUPS
}

\author{
by M. H. ALBERT* and J. LAWRENCE $\dagger$
}

(Received 10th December 1984)

The Ehrenfeucht Conjecture [5] states that if $\mathbb{M}$ is a finitely generated free monoid with nonempty subset $S$, then there is a finite subset $T \subset S$ (a "test set") such that given two endomorphisms $f$ and $g$ on $\mathbb{M}, f$ and $g$ agree on $S$ if and only if they agree on $T$. In [4], the authors prove that the above conjecture is equivalent to the following conjecture: a system of equations in a finite number of unknowns in $\mathbb{M}$ is equivalent to a finite subsystem. Since a finitely generated free monoid embeds naturally into the free group with the same number of generators, it is natural to ask whether a free group of finite rank has the above property on systems of equations. A restatement of the question motivates the following.

Definition. A group $G$ will be said to satisfy the descending chain conditions on solution sets for equations in $k$ variables (denoted $D C C(k)$ ) if for all sequences of group words on a fixed set of $k$ variables, say $w_{1}=w_{1}\left(x_{1}, \ldots, x_{k}\right), w_{2}=w_{2}\left(x_{1}, \ldots, x_{k}\right), \ldots$ there exists only finitely many $l$ such that the solution set of the system $w_{1}=1, w_{2}=1, \ldots$, $w_{l}=1$ is strictly larger than the solution set of the system $w_{1}=1, \ldots, w_{l+1}=1$.

Our question now becomes: does a non-Abelian free group satisfy $D C C(k)$ for all positive integers $k$ ? An affirmative answer to this question would yield an affirmative answer to the Ehrenfeucht Conjecture while a negative answer to this question would suggest a negative answer to the Ehrenfeucht Conjecture. See [10] for an interesting relation between systems of equations in the free monoid and systems of equations in the free group.

Clearly if $H$ is a subgroup of $G$ and $G$ satisfies $D C C(k)$, then $H$ satisfies $D C C(k)$.

In this paper we look at the $D C C(k)$ property in various groups and obtain some results for the free group which partially answer the above question.

Theorem 1. Let $\mathbb{V}$ be a variety of groups and let $\mathbb{F}_{k}$ denote the $k$-generated (relatively) free group in $\mathbb{V}$. The following are equivalent.

(1) The ascending chain condition on normal subgroups holds in $\mathbb{F}_{k}$.

(2) All groups in $\mathbb{V}$ satisfy $D C C(k)$.

Proof. We first show that (1) implies (2).

* Research supported by an NSERC Postdoctoral Fellowship.

$\dagger$ Research partially supported by a grant from NSERC. 
Let $\mathbb{A}_{k}$ denote the $k$-generated free group and suppose that we have a group $G \in \mathbb{V}$ and a sequence of elements of $A_{k}$, say $w_{1}=w_{1}\left(x_{1}, \ldots, x_{k}\right), w_{2}=w_{2}\left(x_{1}, \ldots, x_{k}\right), \ldots$ such that we do not have the descending chain condition on this system. Suppose in particular that we have $\left(a_{i 1}, a_{i 2}, \ldots, a_{i k}\right) \in G$ which is a solution to $w_{j}=1$ for $j=1,2, \ldots, l_{i}$ but is not a solution to $w_{l_{i}+1}=1, i=1,2, \ldots$. The map $\alpha_{i}$ from $A_{k}$ to $G$ sending $x_{j}$ to $a_{i j}$ factors naturally through $\mathbb{F}_{k}$ with $\beta: \mathbb{A}_{k} \rightarrow \mathbb{F}_{k}$ and $\gamma_{i}=\mathbb{F}_{k} \rightarrow G$. Now $\beta\left(w_{j}\left(x_{1}, \ldots, x_{k}\right)\right), j=1,2, \ldots, l_{i}$ are all in the kernel of $\gamma_{i}$ whereas $\beta\left(w_{l_{i}+1}\left(x_{1}, \ldots, x_{k}\right)\right)$ is not. It follows that the normal subgroup of $\mathbb{F}_{k}$ generated by $\left\{\beta\left(w_{j}\left(x_{1}, \ldots, x_{k}\right)\right)\right\}, j=1,2, \ldots, l_{i}$ does not contain $\beta\left(w_{l_{i}+1}\left(x_{1}, \ldots, x_{k}\right)\right)$; hence, we have an infinite ascending chain of normal subgroups of $\mathbb{F}_{k}$.

We now prove that (2) implies (1). Let $\beta$ denote the natural map from the $k$-generated free group $\mathbb{A}_{k}$ to $\mathbb{F}_{k}$ sending generators to generators. Suppose that $N_{1} \subseteq N_{2} \subseteq \ldots$ is an infinite ascending chain of normal subgroups of $\mathbb{F}_{k}$. For each positive integer $l$ choose $w_{l} \in \mathbb{F}_{k}$ such that $\beta\left(w_{l}\right) \in N_{l+1} \backslash N_{l}$. Let $G=\prod_{l=1}^{\infty} \mathbb{F}_{k} / N_{l}$. By construction the system of equations $\left\{w_{l}=1\right\}_{l=1}^{\infty}$ is not equivalent to a finite subsystem. This completes the proof of the theorem.

It should be noted that in the above theorem, if $\mathbb{F}_{k}$ does not satisfy the ascending chain condition on normal subgroups, then we can find a countable group in the variety which does not satisfy $D C C(k)$.

Corollary 2. A nilpotent group satisfies $D C C(k)$ for all positive integers $k$.

Proof. A finitely generated nilpotent group satisfies the ascending chain condition on subgroups [2, p. 1].

For the (relatively) free groups in a variety we have a "test set condition" equivalent to the descending chain condition on solution sets.

Theorem 3. Let $\mathbb{F}_{k}$ denote the $k$-generated free group in a variety. Suppose that each nonempty subset of $\mathbb{F}_{k}$ has a finite test subset. Then $D C C(k)$ holds in $\mathbb{F}_{k}$. Conversely, if $D C C(2 k)$ holds in $\mathbb{F}_{k}$, then the above test set condition also holds.

Proof. Let $A_{k}$ denote the $k$-generated free group generated by $x_{1}, \ldots, x_{k}$ and let $a_{1}, \ldots, a_{k}$ be a set of generators of $\mathbb{F}_{k}$. Let $\beta$ denote the natural map from $\mathbb{A}_{k}$ to $\mathbb{F}_{k}$. Let $w_{1}, w_{2}, \ldots$ be a sequence of elements of $\mathbb{A}_{k}$. The subset of $S$ of $\mathbb{F}_{k}$ will be the image of $\left\{w_{i}\right\}_{i=1}^{\infty}$ under $\beta$. By hypothesis, the set $S$ has a finite test subset $T=\left\{\beta\left(w_{1}\right), \ldots, \beta\left(w_{t}\right)\right\}$. We claim that a solution to the equations $w_{1}=1, \ldots, w_{t}=1$ is a solution to $w_{i}=1$ for all $i$. If not, suppose that $\left(b_{1}, \ldots, b_{k}\right)$ is a solution to the first $t$ equations but not a solution to $w_{i}=1$ for some $i$. Define $\delta: \mathbb{F}_{k} \rightarrow \mathbb{F}_{k}$ by $a_{j} \rightarrow b_{j}$ and let $\gamma: \mathbb{F}_{k} \rightarrow \mathbb{F}_{k}$ be the map sending all elements to 1 . Now $\left.\gamma\right|_{r}=\left.\delta\right|_{r}$, but $\gamma\left(\beta\left(w_{i}\right)\right) \neq \delta\left(\beta\left(w_{i}\right)\right)$, a contradiction. This completes the first part of the proof.

Suppose that $\mathbb{F}_{k}$ satisfies $D C C(2 k)$. Let $S \subset \mathbb{F}_{k}$ and let $U \subset \mathbb{A}_{k}$ denote the preimage of $S$ under $\beta$. Given $w_{j} \in U$, let $w_{j}\left(y_{1}, \ldots, y_{k}\right)=w_{j}\left(z_{1}, \ldots, z_{k}\right)$ be a group equation, $j=1,2, \ldots$. By hypothesis, this system of equations has a finite equivalent subsystem, say the first $l$ equations. We claim that $T=\left\{\beta\left(w_{1}\right), \ldots, \beta\left(w_{l}\right)\right\}$ is a test set for $S$. Suppose that $f$ and $g$ are endomorphisms of $\mathbb{F}_{k}$ which agree on $T$. If $f$ sends $a_{j}$ to $b_{j}$ and $g$ sends $a_{j}$ to $c_{j}$, then 
$\beta\left(w_{i}\right)\left(b_{1}, \ldots, b_{k}\right)=\beta\left(w_{i}\right)\left(c_{1}, \ldots, c_{k}\right), i=1,2, \ldots, l$. Thus $\beta\left(w_{i}\right)\left(b_{1}, \ldots, b_{k}\right)=\beta\left(w_{i}\right)\left(c_{1}, \ldots, c_{k}\right)$ for all $w_{i} \in U$; hence, $f$ and $g$ agree on $S$.

In contrast to nilpotent groups there exist solvable groups of solvable length 3 which do not satisfy $D C C(2)$ In [3] there is an example of a 2-generated, solvable (length 3) non-Hopfian group. Such a group cannot satisfy the ascending chain condition on normal subgroups and so neither can the 2-generated free group in the variety generated by the group. This provides us with the example.

We now strengthen Corollary 2 for certain Abelian groups.

Theorem 4. The following conditions are equivalent for an Abelian group G.

(1) For each positive integer $k$ there is a positive integer $\gamma(k)$ such that a strictly descending chain of solution sets of equations in $k$ variables has length at most $\gamma(k)$.

(2) $G$ is isomorphic to a direct product of a torsion-free Abelian group and direct product of finite cyclic groups each of order less than $N$ for some fixed integer $N$.

Proof. In fact, as we will see, the existence of $\gamma(1)$ is enough to ensure (2). By considering the equations $x^{n !}=1, x^{(n-1) !}=1, \ldots, x=1$, we can see that if $\gamma(1)$ exists, then $G$ is a group of bounded order. We use [9, Theorem 6 and Theorem 8] to complete the proof.

If $G$ has the property described in (2), we look at the torsion-free and torsion part separately. A torsion-free Abelian group can be embedded into a rational vector space and a descending chain of solution sets has length at most $k$. On the other hand, the torsion subgroup of $G$ can be embedded into a direct product of copies of a finite Abelian group. Since a solution set of the direct product is the direct product of a solution set in the finite group, we have the desired result.

We now give several results for free groups.

Theorem 5. A free group satisfies $D C C(2)$. The maximal length of a strictly descending chain of solution sets of equations in 2 variables is 3.

Proof. Let $A_{2}$ be the free group generated by $x$ and $y$ and let $A$ be any free group. If $w(x, y)$ is a non-trivial word in $\mathbb{A}_{2}$ and $(a, b)$ is a solution in $A$, then the image of $A_{2}$ under the map sending $x$ to $a$ and $y$ to $b$ has rank at most 1 [8, Proposition 2.12]. We are now in an infinite cyclic group, and here a descending chain of solution sets has length at most 2 . This completes the proof of the theorem.

In a non-Abelian free group, the system of equations $1=1, x y x^{-1} y^{-1}=1$, $x y^{-1}=1, x=1$ has a strictly descending chain of solutions sets and this shows that the length 3 can actually occur.

Theorem 6. A non-Abelian free group satisfies $D C C(k)$ if and only if the $k$-generated free group, $A_{k}$, satisfies the ascending chain condition on normal subgroups $N$ such that $A / N$ is residually free. 
Proof. Suppose $\mathbb{A}_{k}$ has normal subgroups $N_{1} \varsubsetneqq N_{2} \subsetneq N_{3} \varsubsetneqq \ldots$ such that $\mathbb{A}_{k} / N_{l}$ is residually free for all $l$. Choose $w_{i}\left(x_{1}, \ldots, x_{k}\right) \in N_{i}-N_{i-1}$ and $\gamma_{i}: \mathbb{A}_{k} / N_{i-1} \rightarrow \mathbb{A}$ (A nonAbelian free such that $\gamma_{i}\left(w_{i} N_{i-1}\right) \neq 1$ (since any non-Abelian free group is embedded in any other, we may take a common codomain for all the $\left.\gamma_{i}\right)$. Suppose $\gamma_{i}\left(x_{j} N_{i-1}\right)=a_{j}$ $1 \leqq j \leqq k$. Then, $w_{i}\left(a_{1}, \ldots, a_{k}\right) \neq 1$, but $w_{1}\left(a_{1}, \ldots, a_{k}\right)=\cdots=w_{i-1}\left(a_{1}, \ldots, a_{k}\right)=1$. Thus a non-Abelian free group does not satisfy $D C C(k)$.

Suppose a non-Abelian free group $A$ does not satisfy $D C C(k)$ and let $w_{1}, w_{2}, \ldots$ be a sequence of polynomials with a strictly descending chain of solutions sets. Let $\Phi_{l}=$ $\left\{\phi: \mathbb{A}_{k} \rightarrow \mathbb{A} ; \phi\left(w_{i}\right)=1 i=1, \ldots, l\right\}$. Let

$$
N_{l}=\bigcap_{\phi \in \Phi_{l}} \operatorname{Ker} \phi
$$

Then, $w_{1}, w_{2}, \ldots, w_{l} \in N_{l}, w_{l+1} \notin N_{l}$, so the $N_{l}$ form a strictly increasing chain of normal subgroups. Moreover, $\mathbb{A}_{k} / N_{l}$ is a subdirect product of the $\phi\left(\mathbb{A}_{k}\right) \subseteq \mathbb{A}, \phi \in \Phi_{l}$, and as subgroups of free groups are free this shows $\mathbb{A}_{k} / N_{l}$ is residually free. Thus the proof is complete.

A group $G$ is said to be fully residually free [1] if for each finite subset $S$ of $G-\{1\}$, there is a normal subgroup $N$ of $G$ such that $N \cap S=\emptyset$ and $G / N$ is free. The group $\left\langle a, b, c, d: a^{2} b^{2} c^{2} d^{2}=1\right\rangle$ is an example of a fully residually free group that is not free $[1$, p. 414]. The direct product of two free groups is an example of a residually free group that is not fully residually free [1, p. 404].

Theorem 7. A fully residually free group that is not free cannot be embedded into a finite direct product of free groups.

Proof. Suppose that to the contrary, $G$ is a fully residually free group with normal subgroups $N_{1}, \ldots, N_{l}$ such that $G / N_{i}$ is free and $\bigcap_{i=1}^{l} N_{i}=\langle 1\rangle$. We may suppose, without loss of generality, that $N=\bigcap_{i=1}^{l-1} N_{i} \neq\langle 1\rangle$ and $N_{l} \neq\langle 1\rangle$. As $N_{l} \cap N=\langle 1\rangle$, each element in $N_{l}$ commutes with each element in $N$. By [1, Theorem 1], $N$ is Abelian; hence it is contained in the centre of $G[1$, Lemma 1]. By a second use of [1, Theorem $1]$, we see that this cannot happen in a non-Abelian fully residually free group. This contradiction completes the proof of the theorem.

Corollary 8. The 4-generated free group, $\mathbb{A}_{4}$, does not satisfy the descending chain condition on normal subgroups $N$ such that $\mathbb{A}_{4} / N$ is residually free.

Proof. Let $\alpha$ be the natural map from $A_{4}$ to $G=\left\langle a, b, c, d: a^{2} b^{2} c^{2} d^{2}=1\right\rangle$ and let $\beta$ be the embedding of $G$ into a direct product of free groups. If we let $\gamma_{i}$ denote the projection from the direct product onto the direct product of the first $i$ factors, then the kernels of the maps $\gamma_{i} \circ \beta \circ \alpha$ form, by Theorem 7, an infinite descending chain of normal subgroups of the desired kind.

We conclude this paper by constructing, for each positive integer $l$, an independent system in a non-Abelian free group, of $l$ equations in 3 variables. By independent we mean that for each equation there is a non-solution in the free group which is a solution to the remaining $l-1$ equations. 
Let $\left[a_{1}, a_{2}, \ldots, a_{l}\right]=\left[\left[\ldots\left[\left[a_{1}, a_{2}\right], a_{3}\right] \ldots\right]\right]$ denote the generalized commutator. Let $v_{n}=v_{n}(x, y, z)=z^{-n} x^{n} y^{-1} z^{n}, \quad n=1,2, \ldots$. Define $w_{1}=w_{1}(x, y, z)=\left[v_{2}, v_{3}, \ldots, v_{l}\right], \quad w_{j}=$ $w_{j}(x, y, z)=\left[v_{1}, v_{2}, \ldots, v_{j-1}, v_{j+1}, \ldots, v_{l}\right]$ for $2 \leqq j<l$, and $w_{l}=w_{l}(x, y, z)=\left[v_{1}, v_{2}, \ldots, v_{l-1}\right]$. Let $b$ and $c$ be among a set of generators of a non-Abelian free group $A$. Then $\left(b, b^{j}, c\right)$ is a solution to $w_{i}=1$ if $i \neq j$ but it is not a solution to $w_{j}=1$.

The above example shows that even if a non-Abelian free group satisfies $D C C(3)$ there can be no uniform bound on the length of a strictly descending chain of solution sets, as there is for systems of equations in 2 variables (Theorem 5).

Remark. Since this paper was submitted, the authors have succeeded in proving Ehrenfeucht's Conjecture. The proof makes use of Theorem 1 and some basic properties of metabelian groups. The proof will appear in Theoretical Computer Science. Further results on systems of equations in free and nilpotent groups will appear in a forthcoming paper.

\section{REFERENCES}

1. B. Baumslag, Residually free groups, Proc. London Math. Soc. (3) 17 (1967), 402-418.

2. G. Baumslag, Lecture Notes on Nilpotent Groups (CBMS Series Number 2, Amer. Math. Soc. 1969).

3. G. Baumslag and D. Solitar, Some two generator one relator non-Hopfian groups, Bull. Amer. Math. Soc. 68 (1962), 199-201.

4. K. CUlik II and J. Karhumaki, Systems of equations over a free monoid and Ehrenfeucht's Conjecture, Discrete Math. 43 (1983), 139-153.

5. K. Culik II and A. SalomaA, Test sets and checking words for homomorphism equivalence, J. of Computers and System Sciences 20 (1980), 379-395.

6. S. M. Gersten, On fixed points of automorphisms of finitely generated free groups, Bull. Amer. Math. Soc. 8 (1983), 451-454.

7. R. Lyndon, Equations in groups, Bol. Soc. Brasil Mat. 11 (1980), 79-102.

8. R. Lyndon and P. SchupP, Combinatorial Group Theory (Springer-Verlag, 1977).

9. I. Kaplansky, Infinite Abelian groups (U. of Michigan Press, Ann Arbor, 1969).

10. G. S. Makanin, Systems of equations in free groups, Siberian Math. J. 13 (1972), 402-408.

Department of Pure Mathematics

UNIVERSITY OF WATERLOO

CANADA 